\title{
TEICHMÜLLER SPACES OF RIEMANN SURFACES WITH ORBIFOLD POINTS OF ARBITRARY ORDER AND CLUSTER VARIABLES
}

\author{
LEONID CHEKHOV $*, \dagger$ AND MICHAEL SHAPIRO $\diamond$
}

\begin{abstract}
We define a new generalized class of cluster type mutations for which exchange transformations are given by reciprocal polynomials. In the case of second-order polynomials of the form $x+2 \cos \pi / n_{o}+x^{-1}$ these transformations are related to triangulations of Riemann surfaces of arbitrary genus with at least one hole/puncture and with an arbitrary number of orbifold points of arbitrary integer orders $n_{o}$. In the second part of the paper, we propose the dual graph description of the corresponding Teichmüller spaces, construct the Poisson algebra of the Teichmüller space coordinates, propose the combinatorial description of the corresponding geodesic functions and find the mapping class group transformations thus providing the complete description of the above Teichmüller spaces.
\end{abstract}

\section{INTRODUCTION}

Since their appearance, cluster variables [15] find applications in geometry. An important example of the cluster variables is provided by $\lambda$-lengths [25, 24] of curves that partitions Riemann surfaces with punctures into ideal triangles. In this case, exchange polynomials are quadratic. These coordinates were generalized in [9], 10] to the case of Riemann surfaces with holes. At the same time, a combinatorial description of geodesic functions in terms of the dual variables, the shear coordinates, as well as their quantization, was developed in [5]. Amazingly enough, transition from punctures to holes does not effectively change the corresponding cluster algebra. Generalizations of Teichmüller spaces of Riemann surfaces to the case of bordered Riemann surfaces 22] or ciliated Riemann surfaces [11] were constructed. The corresponding cluster algebras were developed in 20, 12, 13, whereas the geometrical pattern underlying the bordered Riemann surfaces was identified with that of Riemann surfaces with $\mathbb{Z}_{2}$-orbifold points in [2], 3], where the corresponding mutations (flips) in terms of the shear coordinates were constructed. These flips preserve the sets of geodesic functions; the corresponding transformations for cluster variables were considered in 14 and the corresponding mutations were again given by the standard two-term relations. In [6], the description of Teichmüller spaces of Riemann surfaces with holes and with orbifold points of order two and three was given.

*Steklov Mathematical Institute and Laboratoire Poncelet, Moscow, Russia.

$\dagger$ School of Mathematics, Loughborough University, UK..

$\diamond$ Mathematical Department, Michigan State University, East Lansing, USA. 
In the present paper we provide the combinatorial description of Riemann surfaces with holes and with orbifold points of arbitrary orders. We show that mutations for orbifold points of order greater than two are given by three-term transformations (unlike the two-term transformations for order two) determined by a second-order reciprocal polynomial. We prove the Laurent phenomenon and positivity property for these transformations. The positive coefficients of Laurent polynomials however are not necessarily integral in the presence of orbifold points of order greater than three. On the shear-coordinate side, we define the complete set of real-valued coordinates, construct all the geodesic functions for such surfaces, all the mapping-class-group transformations, and prove the regularity condition, that is, that all elements of the corresponding Fuchsian group are hyperbolic or parabolic ones except elements conjugate to loops around orbifold points. We therefore have a regular (up to exactly the indicated orbifold points) Riemann surface with holes for any choice of the introduced real coordinates, and vice versa; these coordinates parametrize therefore the corresponding Teichmüller spaces of Riemann surfaces with holes and with orbifold points of arbitrary orders.

As in the original formulation of cluster algebras, the insight into orbifold triangulations helps us to formulate a more general construction. In [19, particular generalizations of cluster transformations were described that preserve Poisson bracket and have additionally some universal properties. Until recently no applications of these transformations were known. In this paper we compute that mutations of orbifold triangulations are examples of generalization [19]. Another example of generalized quadratic cluster mutations appear under the name quasi-cluster algebra associated with non-orientable surfaces in preprint [8]. Motivated by that we propose a new algebraic construction of generalized cluster algebras with mutations given by reciprocal polynomials of arbitrary order. Using the tools of the standard cluster algebra [16, [17, we prove that the Laurent phenomenon holds true in this case as well. For algebras of order greater than two, we do not know whether the positivity property holds in general; it however holds in all tested examples, so we formulate it as a conjecture. We also prove that generalized cluster algebras of finite type satisfy the same Cartan-Killing classification as the standard cluster algebras. Suggested construction is a particular case of more general construction of [21]. However, we note that generally speaking mutations in [21] preserve neither presymplectic 2-form nor the Poisson bracket.

Acknowledgements. The authors are grateful to Anna Felikson, Pavel Tumarkin, Sergey Fomin, and Dylan Thurston for many enlighting conversations and, specially, to Alek Vainshtein for valuable comments improving our paper.

The work of L.Ch. was supported in part by the Russian Foundation for Basic Research (Grant Nos. 11-01-00440-a and 11-01-12037-ofi-m-2011), by the Grant of Supporting Leading Scientific Schools of the Russian Federation NSh-4612.2012.1, and by the Program Mathematical Methods for Nonlinear Dynamics.

Michael Shapiro was supported in part by grants DMS-0800671 and DMS-1101369.

\section{Generalized Cluster algebra}

We briefly remind the definition of cluster algebra.

An integer $n \times n$ matrix $B$ is called skew-symmetrizable if there exists an integer diagonal $n \times n$ matrix $D=\operatorname{diag}\left(d_{1}, \ldots, d_{n}\right)$, such that the product $B D$ is a skewsymmetric matrix, i.e., $b_{i j} d_{j}=-b_{j i} d_{i}$. 
Let $\mathbb{P}$ be a semi-field equipped with commutative multiplication $\cdot$ and addition $\oplus$. We assume that the multiplicative group of $\mathbb{P}$ is a free abelian group. $\mathbb{P}$ is a coefficient group of cluster algebra. $\mathbb{Z P}$ is the integer group ring, $\mathcal{F}$ is a field of rational functions in $n$ independent variables with coefficients in the field of fractions of $\mathbb{Z P}$. $\mathcal{F}$ is called an ambient field.

Definition 2.1. A seed is a triple $(\mathbf{x}, \mathbf{p}, B)$, where

- $\mathbf{p}=\left(p_{x}^{ \pm}\right)_{x \in \mathbf{x}}$, a $2 n$-tuple of elements of $\mathbb{P}$ is a coefficient tuple of cluster $\mathbf{x}$;

- $\mathbf{x}=\left\{x_{1}, \ldots, x_{n}\right\}$ is a collection of algebraically independent rational functions of $n$ variables which generates $\mathcal{F}$ over the field of fractions of $\mathbb{Z} \mathbb{P}$;

- $B$ is a skew-symmetrizable exchange matrix.

The part $\mathbf{x}$ of seed $(\mathbf{x}, \mathbf{p}, B)$ is called cluster, elements $x_{i} \in \mathbf{x}$ are called cluster variables, and $B$ is called exchange matrix.

Definition 2.2 (seed mutation). For any $k, 1 \leq k \leq n$ we define the mutation of seed $(\mathbf{x}, \mathbf{p}, B)$ in direction $k$ as a new seed $\left(\mathbf{x}^{\prime}, \mathbf{p}^{\prime}, B^{\prime}\right)$ in the following way:

$$
\begin{gathered}
b_{i j}^{\prime}= \begin{cases}-b_{i j}, & \text { if } i=k \text { or } j=k ; \\
b_{i j}+\frac{\left|b_{i k}\right| b_{k j}+b_{i k}\left|b_{k j}\right|}{2}, & \text { otherwise. }\end{cases} \\
x_{i}^{\prime}= \begin{cases}x_{i}, & \text { if } i \neq k ; \\
\frac{p_{k}^{+} \prod_{b_{k j}>0} x_{j}^{b_{k j}}+p_{k}^{-} \prod_{b_{k j}<0} x_{j}^{-b_{k j}},}{x_{k}}, & \text { otherwise. }\end{cases} \\
\text { for } i \neq k \quad p_{k}^{\prime \pm}=p_{k}^{\mp} / p_{i}^{\prime-}= \begin{cases}\left(p_{k}^{+}\right)^{b_{i k}} p_{i}^{+} / p_{i}^{-}, & \text {if } b_{i k} \geq 0 ; \\
\left(p_{k}^{-}\right)^{b_{i k}} p_{i}^{+} / p_{i}^{-}, & \text {if } b_{i k} \leq 0 ;\end{cases}
\end{gathered}
$$

We write $\left(\mathbf{x}^{\prime}, \mathbf{p}^{\prime}, B^{\prime}\right)=\mu_{k}((\mathbf{x}, \mathbf{p}, B))$. Notice that $\mu_{k}\left(\mu_{k}((\mathbf{x}, \mathbf{p}, B))\right)=(\mathbf{x}, \mathbf{p}, B)$. We say that two seeds are mutation-equivalent if one is obtained from the other by a sequence of seed mutations. Similarly we say that two clusters or two exchange matrices are mutation-equivalent.

For any skew-symmetrizable matrix $B$ we define initial seed

$$
(\mathbf{x}, \mathbf{p}, B)=\left(\left\{x_{1}, \ldots, x_{n}\right\},\left\{p_{1}^{ \pm}, \ldots, p_{n}^{ \pm}\right\}, B\right),
$$

where $B$ is the initial exchange matrix, $\mathbf{x}=\left\{x_{1}, \ldots, x_{n}\right\}$ is the initial cluster, $\mathbf{p}=\left\{p_{1}^{ \pm}, \ldots, p_{n}^{ \pm}\right\}$is the initial coefficient tuple.

Cluster algebra $\mathcal{A}(B)$ associated with the skew-symmetrizable $n \times n$ matrix $B$ is a subalgebra of $\mathbb{Q}\left(x_{1}, \ldots, x_{n}\right)$ generated by all cluster variables of the clusters mutation-equivalent to the initial seed $(x, B)$.

Cluster algebra $\mathcal{A}(B)$ is called of finite type if it contains only finitely many cluster variables. In other words, all clusters mutation-equivalent to initial cluster contain totally only finitely many distinct cluster variables.

Two most important properties of cluster algebra are Laurent phenomenon [16] and finite type classification [17. More exactly, Laurent phenomenon states that any cluster variable is expressed as a Laurent polynomial in terms of the initial cluster. The remarkable finite type classification claims that cluster algebras of finite type are in one-to-one correspondence with the Dynkin diagrams of finite type. 
2.1. Generalized cluster transformations. Now we introduce more general cluster transformations.

Assume that $B$ is a skew-symmetrizable integer matrix such that all elements in its $k$ th row are divisible by $d_{k}$. Define $\beta_{k j}=b_{k j} / d_{k}$.

Lemma 2.3. Let $B^{\prime}=\mu_{l}(B)$ be obtained from $B$ by mutation in direction $l$. Then, all entries $b_{k j}^{\prime}$ of $k$-th row of $B^{\prime}$ are divisible by $d_{k}$.

Proof. The statement follows immediately from matrix mutation 2.1.

We now fix $d_{k}$ for all $k$ from 1 to $n$ and assume that all elements $b_{k j}$ of $k$ th row of integer skew-symmetrizable matrix $B$ are divisible by $d_{k}$.

For a collection $\mathbf{p}_{i}=\left(p_{i ; 0}, \ldots, i_{i ; d_{i}}\right)$ we define the exchange polynomial $\theta_{i}\left[\mathbf{p}_{i}\right](u, v)=$ $\sum_{\ell=0}^{d_{i}} p_{i ; \ell} u^{\ell} v^{d_{i}-\ell}$ be a polynomial of degree $d_{i}$. The corresponding inhomogeneous polynomial we denote by $\rho_{i}\left[\mathbf{p}_{i}\right]=\rho_{i}\left[\mathbf{p}_{i}\right](t)=\sum_{\ell=0}^{d_{i}} p_{i ; \ell} t^{\ell}$. Note that, $\theta_{i}\left[\mathbf{p}_{i}\right](u, v)=$ $u^{d_{i}} \rho_{i}\left[\mathbf{p}_{i}\right](v / u)$.

We define a generalized seed $q$ of a generalized cluster algebra as a triple $q=(\mathbf{x}(q), \overline{\mathbf{p}(q)}, B(q))$, where $\mathbf{x}=\left(x_{1}(q), \ldots, x_{n}(q)\right)$ is a $n$-tuple of cluster variables in seed $q, \overline{\mathbf{p}(q)}=\left(\mathbf{p}_{1}(q), \ldots, \mathbf{p}_{n}(q)\right)$ is $n$-tuple of coefficient collections $\mathbf{p}_{i}(q), \mathbf{p}_{i}(q)=$ $\left(p_{i ; 0}(q), \ldots, p_{i ; d_{i}}(q)\right)$ is a $d_{i}+1$-tuple of coefficients of $\theta_{i}[q]$, and, finally, $B(q)$ is an exchange $n \times n$ matrix.

Generalized cluster mutations are described by the following formulas:

Exchange matrix is mutated in direction $k$ by the rule 2.1, which therefore depends only on the degree of the exchange polynomial and not on its coefficients. We introduce $u_{j ;>0}=\prod_{\beta_{j, \ell}>0} x_{\ell}^{\beta_{\ell}}, u_{j ;<0}=\prod_{\beta_{j, \ell}<0} x_{\ell}^{-\beta_{\ell}}$, Mutation of cluster variables is given by the rule $\left(\left\{x_{i}^{\prime}\right\},\left\{\mathbf{p}_{i}^{\prime}\right\}, B^{\prime}\right)=\mu_{k}\left(\left\{x_{i}\right\},\left\{\mathbf{p}_{i}\right\}, B\right)$ :

$$
x_{i}^{\prime}= \begin{cases}x_{i}, & \text { if } i \neq k ; \\ \frac{\theta_{k}\left(u_{k ;>0}, u_{k ;<0}\right)}{x_{k}}, & \text { otherwise. }\end{cases}
$$

Coefficients mutate by the following generalized rule:

$$
\begin{aligned}
p_{k ; \ell}^{\prime} & =p_{k ; d_{k}-\ell} \\
\text { for } i \neq k \quad p_{i ; j}^{\prime} / p_{i ; 0}^{\prime} & = \begin{cases}\left(p_{k ; d_{k}}\right)^{j \beta_{i k}} p_{i ; j} / p_{i ; 0}, & \text { if } b_{i k} \geq 0 ; \\
\left(p_{k ; 0}\right)^{j \beta_{i k}} p_{i ; j} / p_{i ; 0}, & \text { if } b_{i k} \leq 0 ;\end{cases}
\end{aligned}
$$

Remark 2.4. Note that if we assume that coefficients of all $\theta_{i}$ do not change under mutation then the corresponding inhomogeneous polynomial $\rho_{i}$ is reciprocal of degree $d_{i}$, i.e., $t^{d_{i}} \rho(1 / t)=\rho(t)$.

Theorem 2.5. (Laurent property) Any generalized cluster variable is a Laurent polynomial in initial cluster variables $x_{i}$.

Proof. The proof uses the "caterpillar lemma" [16].

Lemma 2.6. Assume that a generalized exchange pattern on $T_{n, m}$ satisfies the following conditions:

(1) For any edge the polynomial $P$ does not depend on $x_{k}$ and is not divisible by any $x_{i}, i \in[n]$.

(2) Each exchange polynomial has nonnegative coefficients 
(3) For any three edges labeled by $i, j, i$

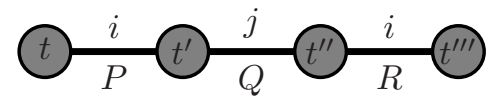

we have $L \cdot Q_{0}^{b} \cdot P=\left.R\right|_{x_{j} \leftarrow \frac{Q_{0}}{x_{j}}}$, where $b$ is a negative integer, $Q_{0}=\left.Q\right|_{x_{i}=0}$, and $L$ is a Laurent monomial whose coefficient lies in $\mathbb{A}$ and is coprime with $P$.

Then each element $x_{i}(t)$ for $i \in[n], t \in T_{n, m}$ is a Laurent polynomial in $x_{1}\left(t_{0}\right), \ldots, x_{n}\left(t_{0}\right)$ with coefficients in $\mathbb{A}$.

By definition of generalized cluster transformation $P=\theta_{i}[t], Q=\theta_{j}\left[t^{\prime}\right], R=$ $\theta_{i}\left[t^{\prime \prime}\right]$.

Note that parts (1) and (2) are evidently satisfied by generalized cluster mutations. It remains to proof part (3). If $x_{i}$ is not included into any monomial of $Q$ then generalized mutation with labels $i$ and $j$ commute and the latter mutation is inverse to the former, namely, $x_{i}\left(t^{\prime \prime \prime}\right)=x_{i}(t)$.

We consider the case where $x_{i}$ enters a monomial of $Q$. For simplicity we denote $b_{r s}\left(t^{\prime}\right)\left(\beta_{r s}\left(t^{\prime}\right)\right)$ by $b_{r s}^{\prime}\left(\beta_{r s}^{\prime}\right.$, resp.) and, specifically, $b_{j i}\left(t^{\prime}\right)$ by $a$.

By our assumptions $a \neq 0$. Moreover, without loss of generality we can assume that $a>0$, otherwise we replace $B$ by $-B$. Since $Q$ is determined by the homogeneous polynomial of two variables where only one variable contains a positive power of $x_{i}$ then $x_{i}$ enters all monomial of $Q$ but one. Hence, $Q_{0}=\left.Q\right|_{x_{i}=0}$ is a monomial. Moreover, $Q_{0}=p_{j ; 0} \prod_{b_{j k}^{\prime}<0} x_{k}^{-b_{j k}^{\prime}}$. Note that $P=\theta_{i}\left(u_{>0}\left(t^{\prime}\right), u_{<0}\left(t^{\prime}\right)\right)$. By the mutation rule 2.2,

$$
b_{i l}\left(t^{\prime \prime}\right)= \begin{cases}b_{i l}^{\prime}, & \text { if } b_{j l}^{\prime} \geq 0 \\ b_{i l}^{\prime}\left(t^{\prime}\right)-b_{i j}^{\prime} b_{j l}^{\prime}, & \text { otherwise. }\end{cases}
$$

By the definition of generalized cluster transformation $R=\theta_{i}\left(u_{>0}\left(t^{\prime \prime}\right), u_{<0}\left(t^{\prime \prime}\right)\right)$.

For $q=t^{\prime}$ or $t^{\prime \prime}$ introduce $\tau_{q}=\prod_{\ell} x_{\ell}(q)^{\beta_{i \ell}(q)}=\frac{u_{>0}(q)}{u_{<0}(q)}$.

Finally,

$$
\begin{aligned}
& \left.\tau_{t^{\prime \prime}}\right|_{x_{j} \leftarrow \frac{Q_{0}}{x_{j}}}=\prod_{l \neq j} x_{l}^{\beta_{i l}^{\prime}} \cdot\left(\frac{Q_{0}}{x_{j}}\right)^{-\beta_{i j}^{\prime}} \prod_{\beta_{j l}\left(t^{\prime}\right)<0} x_{l}^{-b_{i j}^{\prime} b_{j l}^{\prime} / d_{i}}= \\
& \quad=\prod_{l} x_{l}^{\beta_{i l}(t)}\left(p_{j ; 0} \prod_{b_{j l}^{\prime}<0} x_{l}^{-b_{j l}^{\prime}}\right)^{-b_{i j}^{\prime} / d_{i}}\left(\prod_{b_{j l}^{\prime}<0} x_{l}^{-b_{i j}^{\prime} b_{j l}^{\prime} / d_{i}}\right)=p_{j ; 0}^{-\beta_{i j}^{\prime}} \tau_{t^{\prime}}=\frac{1}{p_{j ; 0}^{\beta_{j i j}^{\prime}} \tau_{t}}
\end{aligned}
$$

It is enough to notice that 2.4 and 2.5 imply that $\rho_{t ; i}\left(\tau_{t}\right)=\rho_{t^{\prime \prime} ; i}\left(\left.\tau_{t^{\prime \prime}}\right|_{x_{j} \leftarrow \frac{Q_{0}}{x_{j}}}\right) \cdot L$, where $L$ is a Laurent monomial.

Therefore, $\left.R\right|_{x_{j} \leftarrow \frac{Q_{0}}{x_{j}}}=P \cdot \hat{L}$ where $\hat{L}$ is another Laurent monomial..

Theorem 2.7. Generalized cluster algebras of finite type satisfy the same CartanKilling classification as the standard cluster algebras.

Proof. The proof repeats the one of [17]. The only differences make the proofs of the fact that the only finite type generalized cluster algebras of rank two correspond to $A_{2}, B_{2}, G_{2}$ types. It is checked by direct computation similar to one in [17. Note 
first that in $A_{2}$-case formulas for generalized cluster transformation coincide with formulas for the standard cluster transformation. In $B_{2}$-case the polynomial degrees of theta-polynomials are two and one. Set the theta polynomials in the initial cluster $\theta_{1}(u, v)=a u^{2}+b u v+c v^{2}, \theta_{2}(u, v)=p u+q v$. Then, we immediately obtain $(x, y) \stackrel{\mu_{1}}{\longleftrightarrow}\left(\mu_{1}\right)\left(x_{1}, y\right) \stackrel{\mu_{2}}{\longleftrightarrow}\left(x_{1}, y_{1}\right) \stackrel{\mu_{1}}{\longleftrightarrow}\left(x_{2}, y_{1}\right) \stackrel{\mu_{2}}{\longleftrightarrow}\left(x_{2}, y_{2}\right) \stackrel{\mu_{1}}{\longleftrightarrow}\left(x, y_{2}\right) \stackrel{\mu_{2}}{\longleftrightarrow}$ $(x, y)$, where

$$
\begin{aligned}
& x_{1}=\left(a+b y+c y^{2}\right) / x \\
& y_{1}=\left(p x+q a+b q y+c q y^{2}\right) / x y \\
& x_{2}=\left(a^{2} q^{2}+2 a p q x+a c q^{2} y^{2}+a b q^{2} y+b p q x y+p^{2} x^{2}\right) / x y^{2}, \\
& y_{2}=(q a+p x) / y .
\end{aligned}
$$

Similar computations lead to the 8-cycle in $G_{2}$-case. Note that degrees of polynomials $\operatorname{deg}\left(\theta_{1}\right)=3, \operatorname{deg}\left(\theta_{2}\right)=1$. We set $\theta_{1}(u, v)=a u^{3}+b u^{2} v+c u v^{2}+d v^{3}, \theta_{2}(u, v)=$ $p u+q v . \quad(x, y) \stackrel{\mu_{1}}{\longleftrightarrow}\left(\mu_{1}\right)\left(x_{1}, y\right) \stackrel{\mu_{2}}{\longleftrightarrow}\left(x_{1}, y_{1}\right) \stackrel{\mu_{1}}{\longleftrightarrow}\left(x_{2}, y_{1}\right) \stackrel{\mu_{2}}{\longleftrightarrow}\left(x_{2}, y_{2}\right) \stackrel{\mu_{1}}{\longleftrightarrow}$ $\left(x_{3}, y_{2}\right) \stackrel{\mu_{2}}{\longleftrightarrow}\left(x_{3}, y_{3}\right) \stackrel{\mu_{1}}{\longleftrightarrow}\left(x, y_{3}\right) \stackrel{\mu_{2}}{\longleftrightarrow}(x, y)$, where

$x_{1}=\left(a+b y+c y^{2}+d y^{3}\right) / x$,

$y_{1}=\left(p x+a q+b q y+c q y^{2}+d q y^{3}\right) / x y$,

$x_{2}=\left(a^{3} q^{3}+2 a^{2} c q^{3} y^{2}+2 a^{2} d q^{3} y^{3}+3 a^{2} p q^{2} x+2 a^{2} b q^{3} y+2 a b d q^{3} y^{4}+3 a p^{2} q x^{2}+\right.$ $4 a b p q^{2} x y+a c^{2} q^{3} y^{4}+2 a c d q^{3} y^{5}+a d^{2} q^{3} y^{6}+3 a c p q^{2} x y^{2}+3 a d p q^{2} x y^{3}+a b^{2} q^{3} y^{2}+$ $\left.2 a b c q^{3} y^{3}+b c p q^{2} x y^{3}+b d p q^{2} x y^{4}+p^{3} x^{3}+b^{2} p q^{2} x y^{2}+2 b p^{2} q x^{2} y+p^{2} c q x^{2} y^{2}\right) / x^{2} y^{3}$, $y_{2}=\left(q^{2} a^{2}+a b q^{2} y+a c q^{2} y^{2}+a d q^{2} y^{3}+2 a p q x+b p q x y+p^{2} x^{2}\right) / x y^{2}$,

$x_{3}=\left(a^{2} d q^{3} y^{3}+a^{2} c q^{3} y^{2}+a c p q^{2} x y^{2}+a^{2} b q^{3} y+2 a b p q^{2} x y+b p^{2} q x^{2} y+a^{3} q^{3}+\right.$ $\left.3 a^{2} p q^{2} x+3 a p^{2} q x^{2}+p^{3} x^{3}\right) / x y^{3}$,

$y_{3}=(p x+a q) / y$.

We note that according to 19 the generalized cluster transformation preserves presymplectic structure compatible with cluster algebra structure. Similarly, the secondary generalized cluster transformation preserves the compatible Poisson bracket.

Theorem 2.8. (19) Poisson structure compatible with a cluster algebra is compatible with the corresponding generalized cluster transformations.

In the next section we describe generalized cluster structure associated with triangulated surfaces with orbifold points.

\section{TeICHMÜller SPACE OF SURFACES WITH HOLES AND ORBIFOLD POINTS OF ARBITRARY ORDER}

We now demonstrate how the above mutations with reciprocal polynomials of the second order appear in the description of Teichmüller spaces of Riemann surfaces of arbitrary genus with nonzero number of holes (punctures) and with an arbitrary number $r$ of orbifold points of arbitrary orders.

3.1. The ideal triangle decompositions of orbifold Riemann surfaces and cluster variables. We now present the geometric pattern underlying the algebraic construction of cluster variables corresponding to orbifold Riemann surfaces.

Particular cases of Riemann surfaces with orbifold points of order 2 and 3 are discussed in [2, 4], and 6]. For relation between skew-symmetrizable cluster algebras of finite mutation type and surfaces with orbifold points see [14]. 


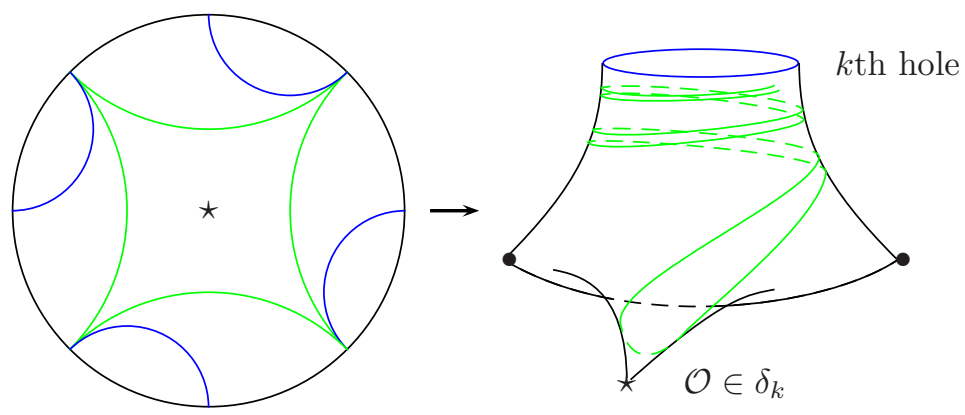

FiguRE 1. The right part represents an example of a regular genus zero Riemann surface contaning a $\mathbb{Z}_{4}$-orbifold point $\mathcal{O}$ marked by a star; the bounding geodesic line is the image of a side of an ideal equilateral square in the Poincaré disc depicted in the left part. Both ends of the geodesic line spiral asymptotically to the closed geodesic that is the boundary of the $k$ th hole.

We consider a regular Riemann surface $\Sigma_{g, s, r}$ of genus $g$ with $s>0$ holes and with a number $r \geq 0$ of orbifold points; orders of these points $p_{i}, i=1, \ldots, r$ are positive integers greater than one.

We introduce the marking on the set of orbifold points splitting this set into nonintersecting (possibly empty) subsets $\delta_{k}, k=1, \ldots, s, \sum_{k=1}^{s}\left|\delta_{k}\right|=r$. For every $k$, we then assign the subset $\delta_{k}$ to the $k$ th hole and introduce a cyclic ordering inside each subset $\delta_{k}$.

To construct the generalization of the ideal triangle decomposition [9, 24, we first remove from the surface all the hyperbolic domains of holes bounded by the corresponding perimeter geodesic lines (with their closures, which are these perimeter lines). Second, we choose for each orbifold point from the set $\delta_{k}$ a domain containing this point and bounded by a geodesic curve whose both ends spiral to the $k$ th hole as shown in the right part of Fig. 1] We remove from the remaining part of the surface all such domains. The remaining part of $\Sigma_{g, s, r}$ admits splitting into ideal triangles; a copy of this splitting can be drawn as a connected ideal polygon in the Poincaré disc; the sides of this polygon are of two sorts: those that are not pre-images of geodesics going around orbifold points must be pairwise identified; to a side that is a pre-image of the geodesics going around $\mathbb{Z}_{p}$ orbifold point we attach (from outside) an equilateral ideal $p$-gone with the orbifold point situated at its geodesic center; this $p$-gone is the $p$-fold covering of the removed domain enclosing the orbifold point.

An example of a fundamental domain of the Riemann surface $\Sigma_{1,1,2}$ of genus one with one hole and with two $\mathbb{Z}_{3}$ orbifold points is in Fig. 2 .

We use the standard geometric correspondence between cluster variables and $\lambda$-lengths: at each point at the absolute that is a vertex of an ideal triangle we set an horocycle; the $\lambda$-length $\ell_{a}$ is then the (signed) geodesic length of the part of side $a$ enclosed between two horocycles based at its endpoints, or the signed distance between horocycles: $\ell_{a}$ is negative when the corresponding horocycles overlap. The

\footnotetext{
${ }^{1}$ In the case of a $\mathbb{Z}_{2}$-orbifold point, this domain has zero area because the corresponding geodesics goes straight to the orbifold point, reflects at it, and repeats its path in the opposite direction.
} 


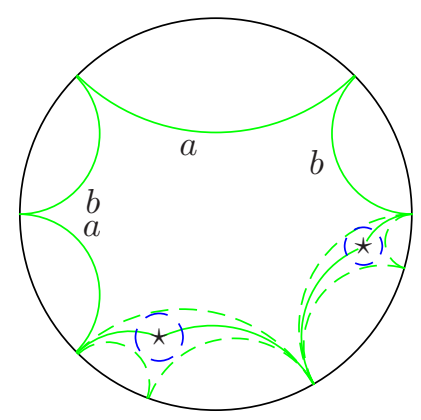

Figure 2. The Poincaré disc with depicted fundamental domain for the genus-one surface $\Sigma_{1,1,2}$ with one hole and two $\mathbb{Z}_{3}$ orbifold points (marked by $\star)$. Solid lines constitute the boundary of a fundamental domain and dashed lines are sides of the related ideal triangles. The sides with labels $a$ and $b$ are pairwise identified. Inscribed circles with centers at the $\mathbb{Z}_{3}$ orbifold points all have the radius $\frac{1}{2} \log 3$.
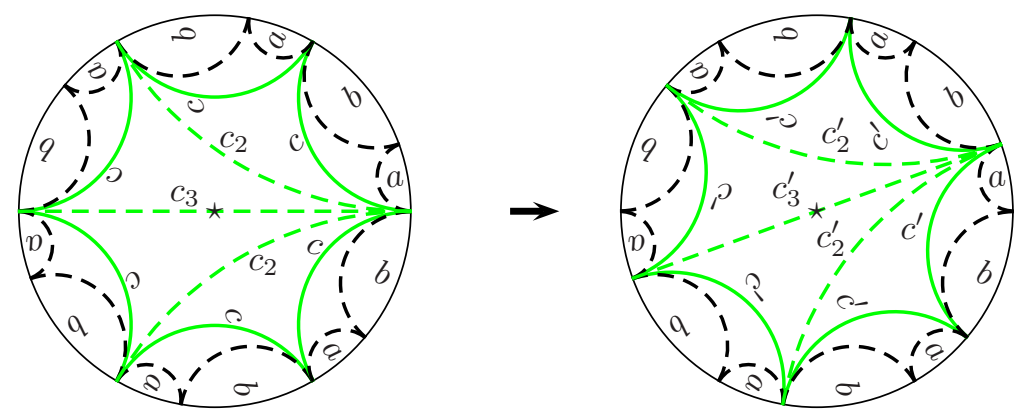

FIGURE 3. The mutation in the ideal $p$-gone (here $p=6$ ), which is the $p$-fold covering of the domain around a $\mathbb{Z}_{p}$-orbifold point (marked by $\star$ ). We must perform a sequence of standard 2-term mutations (3.7) on the set of cluster variables to come from the pattern in the left-hand side to the one in the right-hand side.

correspondence reads

$$
a=e^{\ell_{a} / 2},
$$

and $a$ is the cluster variable associated with the edge.

For an ideal quadrangle with the (cyclically enumerated) sides $a_{i}, i=1, \ldots, 4$ and with diagonals $d$ and $d^{\prime}$ we have the celebrated two-term cluster relation

$$
d d^{\prime}=a_{1} a_{3}+a_{2} a_{4},
$$

which holds independently of the choice of horocycles.

We now consider mutations for cluster variables of the ideal $p$-gone corresponding to a $\mathbb{Z}_{p}$-orbifold point. We consider the pattern in the left-hand side of Fig. 3 and 
perform a sequence of mutations (3.7) to come to the pattern in the right-hand side. For an equilateral $p$-gone, the cluster variable $c_{k}$ for a $k$-diagonal is

$$
c_{k}=c \frac{\sin (\pi k / p)}{\sin (\pi / p)}, \quad\left(c_{1}=c\right) .
$$

The easy combinatorics then yields

Lemma 3.1. $\lambda$-lengths satisfy the following relation

$$
c c^{\prime}=a^{2}+2 \cos (\pi / p) a b+b^{2} .
$$

Remark 3.2. Relation in Lemma 3.1 is a generalized cluster mutation described in Sec. 2. Note that since we use only two-term transformations 3.7 to prove Lemma 3.1, the positivity property for generalized mutations of such form follows from the one for the standard mutations.

To simplify the description, it is convenient to introduce the notion of petal surface. Petals are the domains containing orbifold points that were removed on the second step of constructing the ideal triangulation. To the $k$ th hole, we associate the bouquet of $\left|\delta_{k}\right|$ petals (with no petals if $\delta_{k}$ is empty), each petal carries, besides its cluster variable, the number $\omega_{p}=2 \cos (\pi / p)$. The mutation then occurs inside the corresponding ideal triangle (painted by a light color), and we have three cases depending on whether the adjacent sides are petals themselves:
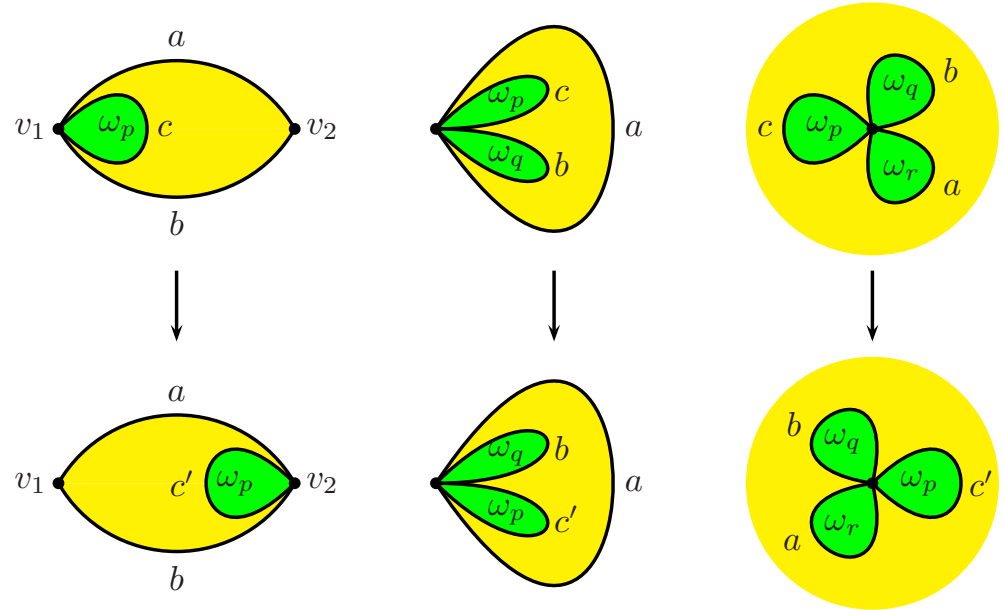

In all the three cases above, the mutation law is given by Lemma 3.1. In the first case, we transfer the cluster variable from one set $\delta_{k}$ to another set (if the vertices $v_{1}$ and $v_{2}$ are distinct); these transformations may also change the cyclic ordering of orbifold points inside a set $\delta_{k}$. Note that the label $\omega_{p}$, being a term of the coefficient tuple, remains assigned to the transformed edge.

3.2. Fat graph description for Riemann surfaces with holes and with $\mathbb{Z}_{p}$ orbifold points. In this subsection and in the rest of the paper, we use the graphs dual to the above ideal triangle decompositions of Riemann surfaces. These graphs are especially useful when describing the Fuchsian groups $\Delta_{g, s, r}$ of Riemann surfaces $\Sigma_{g, s, r}$ and the corresponding geodesic functions.

Definition 3.3. We call a fat graph (a graph with the prescribed cyclic ordering of edges entering each vertex) $\Gamma_{g, s, r}$ a spine of the Riemann surface $\Sigma_{g, s, r}$ with 
$g$ handles, $s>0$ holes, and $r$ orbifold points of the corresponding orders $p_{i}, i=$ $1, \ldots, r$, if

(a) this graph can be embedded without self-intersections in $\Sigma_{g, s, r}$;

(b) all vertices of $\Gamma_{g, s, r}$ are three-valent except exactly $r$ one-valent vertices (endpoints of "pending" edges), which are placed at the corresponding orbifold points;

(c) for an orbifold point from the set $\delta_{k}$, the corresponding pending edge protrudes towards the interior of the face of the graph containing the $k$ th hole; the cyclic ordering of pending edges pointing towards the interior of this face coincide with that of orbifold points in the set $\delta_{k}$;

(d) upon cutting along all edges of $\Gamma_{g, s, r}$ the Riemann surface $\Sigma_{g, s, r}$ splits into $s$ polygons each containing exactly one hole and being simply connected upon gluing this hole.

Edges of this graph are labeled by distinct integers $\alpha=1,2, \ldots, 6 g-6+3 s+2 r$, and we set into the correspondence the real number $Z_{\alpha}$ to each edge.

The first homotopy groups $\pi_{1}\left(\Sigma_{g, s, r}\right)$ and $\pi_{1}\left(\Gamma_{g, s, r}\right)$ coincide because each closed path in $\Sigma_{g, s, r}$ can be homotopically transformed to a closed path in $\Gamma_{g, s, r}$ (taking into account paths that go around orbifold points) in a unique way. The standard statement in hyperbolic geometry is that conjugacy classes of elements of a Fuchsian group $\Delta_{g, s, r}$ are in the 1-1 correspondence with homotopy classes of closed paths in the Riemann surface $\Sigma_{g, s . r}=\mathbb{H}_{+}^{2} / \Delta_{g, s, r}$ and that the "actual" length $\ell_{\gamma}$ of a hyperbolic element $\gamma \in \Delta_{g, s, r}$ coincides with the minimum length of curves from the corresponding homotopy class; it is then the length of a unique closed geodesic line belonging to this class.

When orbifold points are present, the Fuchsian group contains besides hyperbolic elements also elliptic elements corresponding to rotations about these orbifold points. The corresponding generators $\widetilde{F}_{i}, i=1, \ldots, r$, of the rotations through $2 \pi / p_{i}$ are conjugates of the matrices

$$
\widetilde{F}_{i}=U_{i} F_{p_{i}} U_{i}^{-1}, \quad F_{p}=\left(\begin{array}{cc}
0 & 1 \\
-1 & -w
\end{array}\right), \quad w=2 \cos \pi / p, \quad p \geq 2 .
$$

The real numbers $Z_{\alpha}$ in Definition 3.3 are the $h$-lengths 24: they are called the (Thurston) shear coordinates [25, [1 in the case of punctured Riemann surface (without boundary components). We preserve this notation and this term also in the case of orbifold surfaces. These coordinates are related to the cross-ratio relation for two adjacent ideal triangles constituting the ideal quadrangle with the respective vertices (in the cyclic order) $a, b, c, d$ and diagonal $b d$. At the same time, they are related to the cluster variables $a_{i}$ corresponding to the sides of the corresponding quadrangle. We have

$$
e^{Z}=-\frac{(b-c)(d-a)}{(b-a)(d-c)}=\frac{a_{1} a_{3}}{a_{2} a_{4}}
$$

and we obtain the parameter $Z_{\alpha}$ choosing $\{a, b, c, d\}=\left\{-1,0, e^{Z_{\alpha}}, \infty\right\}$.

For example, the fat graph corresponding to the pattern in Fig. 2 is depicted in Fig. 4.

3.3. The Fuchsian group $\Delta_{g, s, r}$ and geodesic functions. We now describe combinatorially the conjugacy classes of the Fuchsian group $\Delta_{g, s, r}$. Every time the 


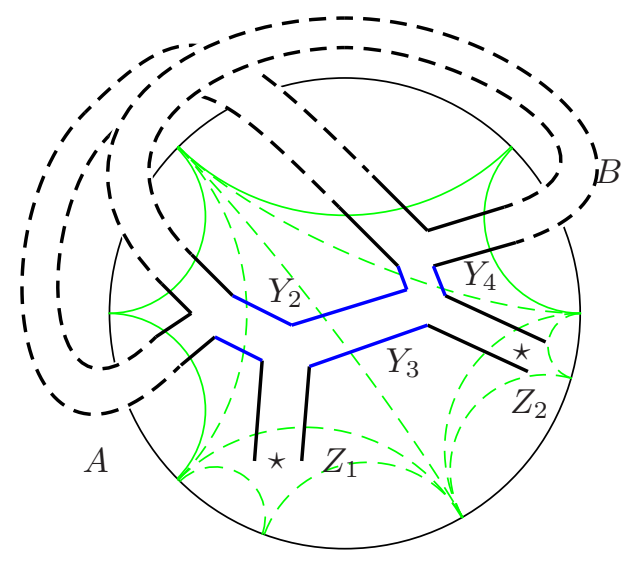

FIGURE 4. The fat graph corresponding to the ideal triangle partition of the fundamental domain in Fig. 2. The real numbers $Z_{1}$ and $Z_{2}$ are associated to the pending edges and $A, B$, and $Y_{2}, Y_{3}$, and $Y_{4}$ to the inner edges.

path homeomorphic to a (closed) geodesic $\gamma$ goes through the edge with the label $\alpha$ we insert [9] the matrix of the Möbius transformation

$$
X_{Z_{\alpha}}=\left(\begin{array}{cc}
0 & -e^{Z_{\alpha} / 2} \\
e^{-Z_{\alpha} / 2} & 0
\end{array}\right) .
$$

We also have the "right" and "left" turn matrices to be set in proper places when a path makes the corresponding turns at three-valent vertices,

$$
R=\left(\begin{array}{cc}
1 & 1 \\
-1 & 0
\end{array}\right), \quad L=R^{2}=\left(\begin{array}{cc}
0 & 1 \\
-1 & -1
\end{array}\right)
$$

New elements of the Fuchsian group correspond to rotations of geodesic lines when going around orbifold points indicated by star-vertices; for a $\mathbb{Z}_{p}$ orbifold point we then insert the matrix $F_{p}(3.9)$ into the corresponding string of $2 \times 2$-matrices (when we go around the orbifold point counterclockwise as in Fig. 5(a)). When the order of the orbifold point is larger than two, we can go around it $k$ times; we then have to insert the matrix $(-1)^{k+1} F_{p}^{k}$ into the product of $2 \times 2$-matrices. For example, parts of geodesic functions in the three cases in Fig. 5 read

$$
\begin{array}{ll}
\text { (a) } & \ldots X_{X} L X_{Z} F_{p} X_{Z} L X_{Y} \ldots \\
\text { (b) } & \ldots X_{X} L X_{Z}\left(-F_{p}^{2}\right) X_{Z} R X_{X} \ldots \\
\text { (c) } & \ldots X_{Y} R X_{Z}\left(F_{p}^{3}\right) X_{Z} L X_{Y} \ldots
\end{array}
$$

Note that $F_{p}^{p}=(-1)^{p-1} \mathbb{E}$, so going around the $\mathbb{Z}_{p}$ orbifold point $p$ times merely corresponds to avoiding this orbifold point due to the simple equality (note that $X_{S}^{2}=-\mathbb{E}$ and $\left.L^{2}=-R\right)$

$$
X_{X} L X_{Z}(-1)^{p-1} F_{p}^{p} X_{Z} L X_{Y}=X_{X} L X_{Z}^{2} L X_{Y}=-X_{X} L^{2} X_{Y}=X_{X} R X_{Y} .
$$

(For the $\mathbb{Z}_{2}$ orbifold points this pattern was first proposed by Fock and Goncharov [1]; the graph morphisms were described in [4.) 


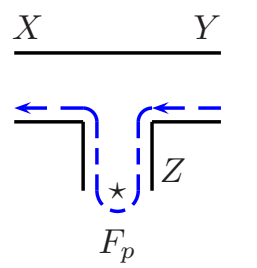

(a)

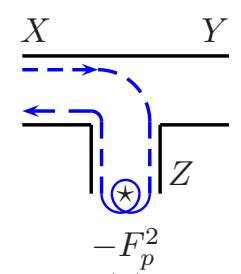

(b)

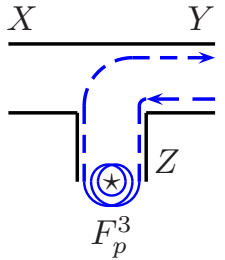

(c)

FIguRE 5. Part of a graph with a pending edge. Its endpoint with the orbifold point is directed toward the interior of the boundary component this point is associated with. The variable $Z$ corresponds to the respective pending edge. We present four typical examples of geodesics undergoing single (a), double (b), and triple (c) rotations at the $\mathbb{Z}_{p}$ orbifold point.

An element of a Fuchsian group has then the typical structure

$$
P_{\gamma}=L X_{Y_{n}} R X_{Y_{n-1}} \cdots R X_{Y_{2}} L X_{Z_{1}}(-1)^{k+1} F_{p}^{k} X_{Z_{1}} R X_{Y_{1}},
$$

where $Y_{i}$ are variables of "internal" edges and $Z_{j}$ are those of pending edges. The corresponding geodesic function

$$
G_{\gamma} \equiv \operatorname{Tr} P_{\gamma}=2 \cosh \left(\ell_{\gamma} / 2\right)
$$

is expressed via the actual length $\ell_{\gamma}$ of the closed geodesic on the Riemann surface.

Remark 3.4. Note that the combinations

$$
R X_{y}=\left(\begin{array}{cc}
e^{-Y / 2} & -e^{Y / 2} \\
0 & e^{Y / 2}
\end{array}\right) \text { and } L X_{y}=\left(\begin{array}{cc}
e^{-Y / 2} & 0 \\
-e^{-Y / 2} & e^{Y / 2}
\end{array}\right)
$$

as well as products of any number of these matrices have the sign structure $\left(\begin{array}{cc}+ & - \\ - & +\end{array}\right)$, so the trace of any of $P_{\gamma}$ in the absence of orbifold points is a sum of exponentials with positive integer coefficients; this sum always include the terms $e^{Y_{1} / 2+\cdots+Y_{n} / 2}$ and $e^{-Y_{1} / 2-\cdots-Y_{n} / 2}$ being therefore always greater or equal two thus describing a hyperbolic or parabolic element; the latter is possible only if $Y_{1}+\cdots+Y_{n}=0$ and only if the turn matrices in (3.14) are all either $R$ or $L$, which corresponds to a path going along the boundary of a face; all such paths are homeomorphic to the hole boundaries, and the condition that the sum of $Y_{i}$ equals zero indicates the degeneration of a hole into a puncture.

The group generated by elements (3.9) together with translations along $A$ - and $B$-cycles and around holes not necessarily produces a regular (metrizable) surface because its action is not necessarily discrete. We formulate the necessary and sufficient conditions for producing a regular surface in terms of graphs (see [3] for the $\mathbb{Z}_{2}$ orbifold point case) 2

To formulate the regularity condition, we interpret passages around orbifold points as paths in the $p$-fold covering of the geodesic neighborhood in Fig. 1 For this, we take the subgraph in Fig. [6 dual to the ideal triangle decomposition in

\footnotetext{
${ }^{2}$ In what follows, we call a Riemann surface regular if it is locally a smooth constant-curvature surface everywhere except exactly $r$ orbifold points.
} 


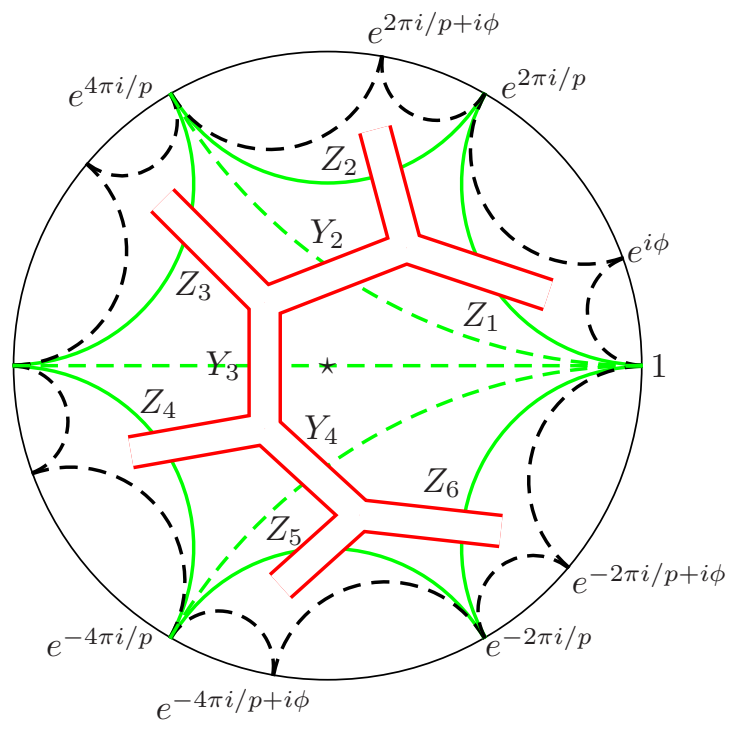

FiguRE 6. The (tree-like) subgraph dual to the ideal triangle decomposition of the ideal equilateral $p$-gone in Fig. 3 . All the variables $Z_{\alpha}$ and $Y_{\beta}$ are determined by the cross-ratio relations in the corresponding ideal quadrangles based on the points from the sets $\left\{e^{2 \pi i k / p}\right\}$ and $\left\{e^{2 \pi i k / p+i \phi}\right\}, k=0, \ldots, p-1$.

Fig. 3. When splitting the equilateral $p$-gone into ideal triangles we break the $p$ fold symmetry, so now the shear coordinates $Z_{i}, i=1, \ldots, p$, on the fat graph edges dual to the corresponding $p$-gone sides and $Y_{j}, j=2, \ldots, p-2$, on the edges dual to the diagonals of the $p$-gone are different.

We first identify the parameter $Z$ in (3.13) to be

$$
e^{Z}=\frac{\sin (\pi / p-\phi / 2)}{\sin (\phi / 2)},
$$

We can then derive the explicit relations between the parameter $Z$ in (3.16) and the variables $Z_{i}$ and $Y_{j}$ determined by the standard cross-ratio relations (3.10). The vertices of the ideal $p$-gone are situated at the points $e^{i 2 \pi k / p}, k=0, \ldots, p-1$, and $p$ copies of the vertex of an additional ideal triangle adjacent to the $p$-gone side are $e^{i \phi+i 2 \pi i k / p}, k=0, \ldots, p-1$.

Using the cross-ratio relations to calculate $Z_{i}$ and $Y_{j}$ we find the exact relations between these variables and the variable $Z$ given by (3.16):

$$
\begin{aligned}
e^{Z_{1}} & =e^{Z} \frac{\sin (2 \pi / p)}{\sin (\pi / p)}, \\
e^{Z_{p}} & =e^{Z} \frac{\sin (\pi / p)}{\sin (2 \pi / p)}, \\
e^{Z_{k}} & =e^{Z} \frac{\sin ((k-1) \pi / p)}{\sin (k \pi / p)}, \quad k=2, \ldots, p-1,
\end{aligned}
$$




$$
e^{Y_{k}}=\frac{\sin ((k+1) \pi / p)}{\sin ((k-1) \pi / p)}, \quad k=2, \ldots, p-2 .
$$

The following $2 \times 2$-matrix equalities can be verified directly:

$$
X_{Z} F_{p} X_{Z}=X_{Z_{1}} L X_{Z_{2}}=X_{Z_{k}} L X_{Y_{k}} L X_{Z_{k+1}}=X_{Z_{p-1}} L X_{Z_{p}}, k=2, \ldots, p-2 .
$$

We then have the following lemma.

Lemma 3.5. We have the following explicit $2 \times 2$-matrix relations for the shear variables in the equilateral p-gone in Fig. (6) given by (3.17) and (3.18):

$$
\begin{aligned}
X_{Z} F_{p} X_{Z} & =X_{Z_{1}} L X_{Z_{2}} \\
X_{Z}\left(-F_{p}^{2}\right) X_{Z} & =X_{Z_{1}} R X_{Y_{2}} L X_{Z_{3}} \\
\vdots & \\
X_{Z}(-1)^{k-1} F_{p}^{k} X_{Z} & =X_{Z_{1}} R X_{Y_{2}} R \cdots R X_{Y_{k}} L X_{Z_{k+1}}, \quad k=2, \ldots, p-2 \\
X_{Z}(-1)^{p} F_{p}^{p-1} X_{Z} & =X_{Z_{1}} R X_{Y_{2}} R \cdots R X_{Y_{p-2}} R X_{Z_{p}} .
\end{aligned}
$$

The proof uses equalities from (3.19) for constructing the longer chain using that $X_{S} X_{S}=-\mathbb{E}$ for any variable $S$ and that $L^{2}=-R$. For example, we obtain the r.h.s. of the second equality in (3.20) multiplying $X_{Z_{1}} L X_{Z_{2}} \cdot X_{Z_{2}} L X_{Y_{2}} L X_{Z_{3}}$ whereas the l.h.s. merely becomes $X_{Z} F_{p} X_{Z} \cdot X_{Z} F_{p} X_{Z}=X_{Z}(-1) F_{p}^{2} X_{Z}$. All other equalities are obtained if we continue this chain of multiplications.

Due to Lemma 3.5, all "rotations" about orbifold points $X_{Z}(-1)^{k+1} F_{p}^{k} X_{Z}$ are now presented as the standard products of matrices $X_{S}$ (with real $S$ ) alternated with the matrices of left and right turns (3.12), which means that the conclusion of Remark 3.4 remains valid in this case as well: as soon as in the original spine $\Gamma_{g, s, r}$ all the parameters $Z_{\alpha}$ of pending edges are real, all the geodesic functions constructed on a Riemann surface with orbifold points are Laurent polynomials with positive integer coefficients of the "new" real variables $Z_{i}^{(\alpha, p)}, Y_{j}^{(p)}$ (where the superscripts $\alpha, p$ indicate that these variables are completely determined by the original variable $Z_{\alpha}$ and the order $p$ of the orbifold point) and "old" variables of "internal" edges of the spine $\Gamma_{g, s, r}$. corresponding to usual partitions into ideal triangles. So, again, in the trace of every product of form (3.14) we necessarily have the term $2 \cosh \left(\sum_{\beta=1}^{n} X_{\beta}\right)$, where the sum ranges all edges (new and internal ones) the corresponding path goes through, and we let $X$ denote the variables of all these edges disregarding their origins. Every such trace is therefore a positive number greater or equal two, and the corresponding element of the group will be either hyperbolic or parabolic (the latter is possible only for geodesics around holes and only if a hole reduces to a puncture). The only elliptic elements are precisely conjugates of $F_{p}^{k}$. We therefore come to the theorem

Theorem 3.6. We have a metrizable Riemann surface for any choice of real numbers $Z_{\alpha}$ associated to the edges of an original spine $\Gamma_{g, s, r}$. The converse statement is also true: for any metrizable Riemann surface $\Sigma_{g, s, r}$ we have a spine $\Gamma_{g, s, r}$ with real numbers associated to its edges such that the lengths of geodesics on $\Sigma_{g, s, r}$ are given by traces of products (3.14) corresponding to paths in the spine.

The proof of the second statement was performed in 4 for $\mathbb{Z}_{2}$-orbifold points. It is based on the (obvious) existence of the ideal triangle decomposition described in 
Sec. 3.1 for any metrizable Riemann surface and can be straightforwardly generalized to the case of orbifold points of any type. We have therefore parameterized all possible regular surfaces in terms of the $(6 g-6+3 s+2 r)$-tuple of real coordinates $\left\{Z_{\alpha}\right\}$.

Corollary 3.7. The decorated Teichmüller space $\mathfrak{T}_{g, s, r}^{H}$ of Riemann surfaces with holes and orbifold points is the space $\mathbb{R}^{6 g-6+3 s+2 r}$ of real parameters on the edges of a spine $\Gamma_{g, s, r}$.

\section{MAPPing Class GROUP TRANSFORMATIONS}

4.1. Poisson structure. One of the most attractive properties of the graph description is a very simple Poisson algebra on the set of parameters $Z_{\alpha}$. The following result is the straightforward generalization of the theorem formulated for surfaces without marked points in 9] and for surfaces with order-2 orbifold points in [1] (see also [2]).

Theorem 4.1. In the coordinates $Z_{\alpha}$ on any fixed spine corresponding to a surface with orbifold points, the Weil-Petersson bracket $B_{W P}$ is given by

$$
\{f(\mathbf{Z}), g(\mathbf{Z})\}=\sum_{\begin{array}{c}
\text { 3-valent } \\
\text { vertices } \alpha=1
\end{array}}^{4 g+2 s+|\delta|-4} \sum_{i=1}^{3}\left(\frac{\partial f}{\partial Z_{\alpha_{i}}} \frac{\partial g}{\partial Z_{\alpha_{i+1}}}-\frac{\partial g}{\partial Z_{\alpha_{i}}} \frac{\partial f}{\partial Z_{\alpha_{i+1}}}\right),
$$

where the sum ranges all the three-valent vertices of a graph and $\alpha_{i}$ are the labels of the cyclically (counterclockwise) ordered $\left(\alpha_{4} \equiv \alpha_{1}\right)$ edges incident to the vertex with the label $\alpha$ irrespectively on whether these edges are internal or pending edges of the graph. This bracket gives rise to the Goldman bracket on the space of geodesic length functions [18.

We identify the exchange matrix $B$ with the matrix of the Poisson relations for the variables $Z_{\alpha}$.

The center of this Poisson algebra is provided by the proposition.

Proposition 4.2. The center of the Poisson algebra (4.21) is generated by elements of the form $\sum Z_{\alpha}$, where the sum ranges all edges of $\Gamma_{g, s, r}$ belonging to the same boundary component taken with multiplicities. This means, in particular, that each pending edge, irrespectively on the type of orbifold point it corresponds to, contributes twice to such sums. The dimension of this center is obviously s.

For the proof in the general case see Appendix B of [7]. Note that for the path homeomorphic to the hole boundary, for any number of insertions of matrices $F_{p_{i}}$ with any $p_{i}$, we have

$$
\begin{aligned}
\operatorname{tr} & {\left[L X_{Y_{1}} L X_{Y_{2}} \cdots L X_{Y_{k}} F_{p_{i}} X_{Y_{k}} L \cdots L X_{Y_{n-1}} L X_{Y_{n}}\right] } \\
& =2 \cosh \left[\frac{Y_{1}}{2}+\frac{Y_{2}}{2}+\cdots+Y_{k}+\cdots+\frac{Y_{n-1}}{2}+\frac{Y_{n}}{2}\right] .
\end{aligned}
$$

4.2. Flip morphisms of fat graphs. In this section, we present the complete list of mapping class group transformations that enable us to change numbers $\left|\delta_{k}\right|$ of orbifold points associated with the $k$ th hole, change the cyclic ordering inside any of the sets $\delta_{k}$, flip any inner edge of the graph and, eventually, change the orientation of the geodesic spiraling to the hole perimeter (in the case where we have more than 


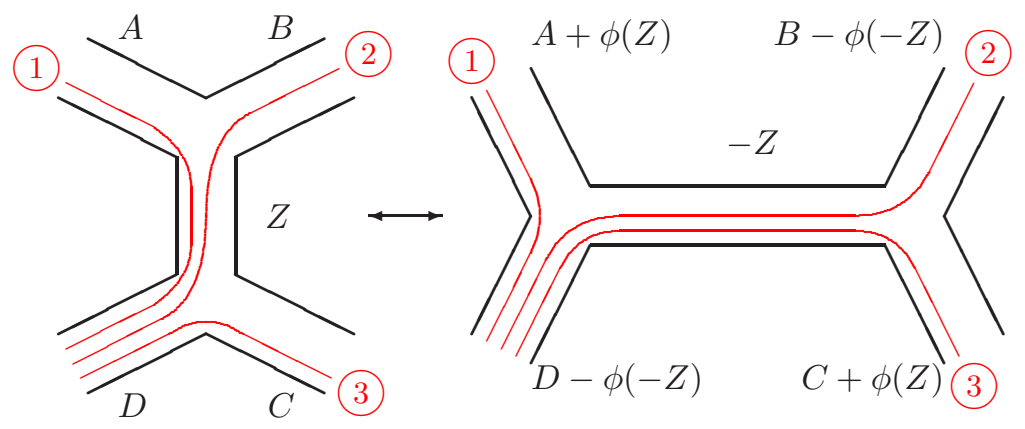

FIGURE 7. Flip, or Whitehead move on the shear coordinates $Z_{\alpha}$. The outer edges can be pending, but the edge with respect to which the morphism is performed must be an internal edge. We also indicate the correspondences between geodesic paths under the flip.

one hole) 3 We can therefore establish a morphism between any two of the graphs belonging to the same class $\Gamma_{g, s, r}$ with the same (unordered) sets of orbifold point orders $\left\{p_{i}\right\}_{i=1}^{r}$.

4.2.1. Whitehead moves on inner edges. Given a spine $\Gamma$ of $\Sigma$ and assuming that the edge $\alpha$ has distinct endpoints, we may produce another spine $\Gamma_{\alpha}$ of $\Sigma$ by contracting and expanding edge $\alpha$ of $\Gamma$, the edge labeled $Z$ in Figure 7 This transformation is dual to the mutation (3.7). We say that $\Gamma_{\alpha}$ arises from $\Gamma$ by a Whitehead move (or flip) along the edge $\alpha$. A labeling of edges of the spine $\Gamma$ implies a natural labeling of edges of the spine $\Gamma_{\alpha}$; we then obtain a morphism between the spines $\Gamma$ and $\Gamma_{\alpha}$.

Proposition 4.3. [5] Setting $\phi(Z)=\log \left(1+e^{Z}\right)$ and adopting the notation of Fig. 7 for shear coordinates of nearby edges, the effect of a Whitehead move is

(4.22) $W_{Z}:(A, B, C, D, Z) \rightarrow(A+\phi(Z), B-\phi(-Z), C+\phi(Z), D-\phi(-Z),-Z)$

In the various cases where the edges are not distinct and identifying an edge with its shear coordinate in the obvious notation we have: if $A=C$, then $A^{\prime}=A+2 \phi(Z)$; if $B=D$, then $B^{\prime}=B-2 \phi(-Z)$; if $A=B$ (or $C=D$ ), then $A^{\prime}=A+Z$ (or $\left.C^{\prime}=C+Z\right)$; if $A=D($ or $B=C)$, then $A^{\prime}=A+Z\left(\right.$ or $\left.B^{\prime}=B+Z\right)$. Any subset of edges $A, B, C$, and $D$ can be pending edges of the graph.

We have the lemma establishing the properties of invariance w.r.t. the flip morphisms [5].

Lemma 4.4. Transformation (4.22) preserves the traces of products over paths (3.15) (the geodesic functions) and transformation (4.22) simultaneously preserves Poisson structure (4.21) on the shear coordinates.

4.2.2. Whitehead moves on pending edges. Choosing other representatives of the orbifold points in the Poincaré disc, we obtain different fundamental domains with different cyclic ordering of the (preimages) of the orbifold points $s_{i}\left(i=1, \ldots,\left|\delta_{k}\right|\right)$ possibly with transferring orbifold points from one set $\delta_{k}$ to another set $\delta_{k^{\prime}}$.

Analogously to the mutation in Fig. 3 flipping the pending edge corresponds to choosing another fundamental domain, as shown in Fig. 8. We take there $e^{Z}$ given

\footnotetext{
${ }^{3}$ These transformations are dual to mutations of cluster variables from Sec. 2
} 


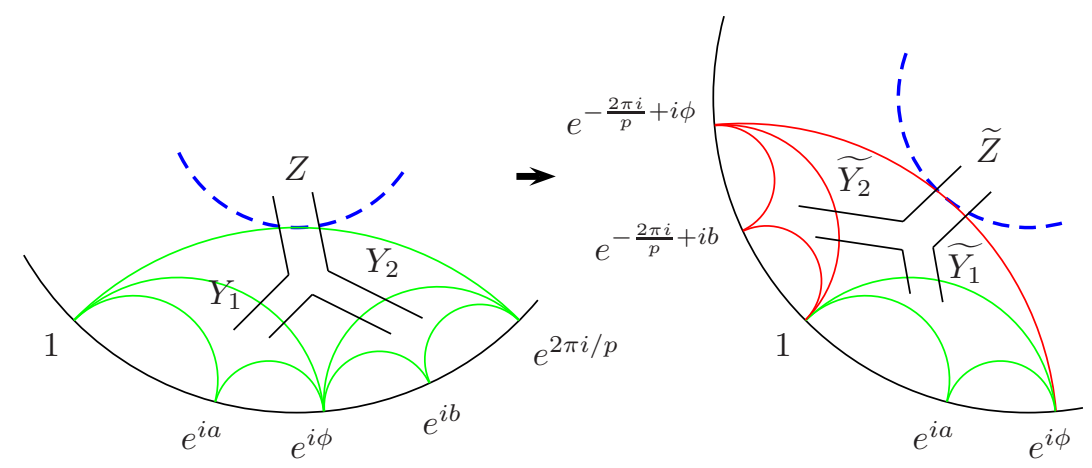

Figure 8. The transformation of dual variables ( $h$-lengths) $\left\{Y_{1}, Y_{2}, Z\right\} \rightarrow\left\{\widetilde{Y_{1}}, \widetilde{Y_{2}}, \widetilde{Z}\right\}$ described by (4.23) with $w=2 \cos (\pi / p)$.

by formula (3.16) and $e^{Y_{1,2}}$ and $e^{\widetilde{Y_{1,2}}}$ given by the standard cross-ratio relations, for example,

$$
e^{Y_{2}}=\frac{\left(1-e^{i \phi}\right)\left(e^{i b}-e^{2 \pi i / p}\right)}{\left(e^{i \phi}-e^{i b}\right)\left(1-e^{2 \pi i / p}\right)}, \quad e^{\widetilde{Y_{2}}}=\frac{\left(e^{-2 \pi i / p+i b}-1\right)\left(e^{-2 \pi i / p+i \phi}-e^{i \phi}\right)}{\left(e^{-2 \pi i / p+i \phi}-e^{-2 \pi i / p+i b}\right)\left(1-e^{i \phi}\right)} .
$$

Lemma 4.5. The transformation in Fig. 8 with $e^{Z}$ given by (3.16) has the form

$$
\left\{\tilde{Y}_{1}, \tilde{Y}_{2}, \tilde{Z}\right\}=\left\{Y_{1}-\log \left(1+w e^{-Z}+e^{-2 Z}\right), Y_{2}+\log \left(1+w e^{Z}+e^{2 Z}\right),-Z\right\}
$$

and is the morphism of the space $\mathcal{T}_{g, s, r}^{H}$. These morphisms preserve both Poisson structures (4.21) and the geodesic functions. In Fig. 8 any (or both) of $Y$-variables can be variables of pending edges (the transformation formula is insensitive to it).

Proof. Verifying the preservation of Poisson relations (4.21) is simple, whereas for traces over paths we have four cases, and in each of these cases we have the following $2 \times 2$-matrix equalities to be verified directly:

$$
\begin{aligned}
& X_{Y_{2}} L X_{Z} F_{p}^{k} X_{Z} L X_{Y_{1}}=-X_{\tilde{Y}_{2}} R X_{\tilde{Z}} F_{p}^{k-1} X_{\tilde{Z}} R X_{\tilde{Y}_{1}}, \\
& X_{Y_{1}} R X_{Z} F_{p}^{k} X_{Z} L X_{Y_{1}}=-X_{\tilde{Y}_{1}} L X_{\tilde{Z}} F_{p}^{k} X_{\tilde{Z}} R X_{\tilde{Y}_{1}}, \\
& X_{Y_{2}} L X_{Z} F_{p}^{k} X_{Z} R X_{Y_{2}}=-X_{\tilde{Y}_{2}} R X_{\tilde{Z}} F_{p}^{k} X_{\tilde{Z}} L X_{\tilde{Y}_{2}} .
\end{aligned}
$$

Using flip morphisms in Fig. 8 and in formula (4.22), we establish a morphism between any two algebras corresponding to surfaces of the same genus, same number of boundary components, and same numbers of $\mathbb{Z}_{p}$-orbifold points of each sort $p$; the distribution of latter into the boundary components as well as the cyclic ordering inside each of the boundary component can be arbitrary.

It is a standard tool that if, after a series of morphisms, we come to a graph of the same combinatorial type as the initial one (disregarding labeling of edges but distinguishing between different orbifold types of pending vertices), we associate a mapping class group operation to this morphism therefore passing from the groupoid of morphisms to the group of modular transformations.

Remark 4.6. Another way of interpreting transformations (4.23) is as follows. We can imitate the above flips/mutations by introducing a new "hole" with possibly imaginary perimeter $P$ and considering the following chain of standard flips: 


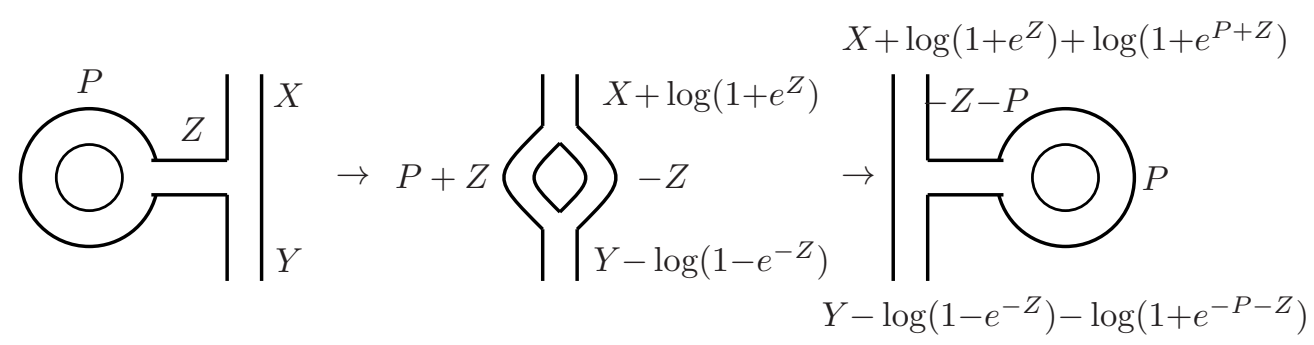

In this pattern, it is useful to shift the variable $Z$ and introduce

$$
\bar{Z}=Z+\frac{P}{2} \text {. }
$$

The transformation for the variables $X$ and $Y$ then just becomes (4.23),

$$
\begin{aligned}
{\left[\begin{array}{l}
X \\
\bar{Z}
\end{array}\right] } & \rightarrow\left[\begin{array}{l}
X+\log \left[\left(1+e^{\bar{Z}-P / 2}\right)\left(1+e^{\bar{Z}+P / 2}\right)\right] \\
Y-\log \left[\left(1+e^{-\bar{Z}+P / 2}\right)\left(1+e^{-\bar{Z}-P / 2}\right)\right] \\
-\bar{Z}
\end{array}\right]= \\
& =\left[\begin{array}{l}
X+\log \left[1+\omega_{p} e^{\bar{Z}}+e^{2 \bar{Z}}\right] \\
Y-\log \left[1+\omega_{p} e^{-\bar{Z}}+e^{-2 \bar{Z}}\right] \\
-\bar{Z}
\end{array}\right],
\end{aligned}
$$

where $\omega_{p}=e^{P / 2}+e^{-P / 2}$.

Since $P$ is not affected by the above sequence of flips, we can merely erase the corresponding loop and present it as flipping the pending edge, which carries besides the cluster variable $\bar{Z}$ also the coefficient $\omega_{p}$, which is preserved by mutations and is equal to $2 \cos (\pi / p)$ in the geometric case.

4.2.3. Changing the spiraling direction. The last mapping class group transformation changes the sign of the hole perimeter:

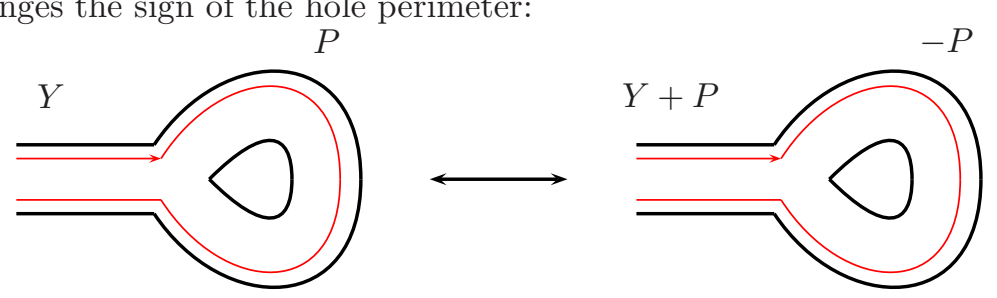

Lemma 4.7. Transformation (4.24) preserves the Poisson brackets and the set of geodesic functions.

Proof. The preservation of the Poisson bracket is obvious because the variable $P$ Poisson commutes with all other variables, whereas the preservation of geodesic functions follows from two matrix equalities:

$$
\begin{aligned}
& X_{Y} L X_{P} L X_{Y}=X_{Y+P} L X_{-P} L X_{Y+P}, \\
& X_{Y} R X_{P} R X_{Y}=X_{Y+P} R X_{-P} R X_{Y+P} .
\end{aligned}
$$

We can therefore enlarge the mapping class group of $\mathcal{T}_{g, s, r}^{H}$ by adding symmetries between sheets of the $2^{s}$-ramified covering of the "genuine" (nondecorated) Teichmüller space $\mathcal{T}_{g, s, r}$. 
The geometrical meaning of this transformation is clear: we change the direction of spiraling to the hole perimeter line for all lines of the ideal triangle decomposition that spiral to a given hole like in Fig. 1

We can summarize as follows.

Theorem 4.8. The whole mapping class group of $\Sigma_{g, s, r}$ is generated by morphisms described by Lemmas 4.4, 4.5, and 4.7.

\section{Conjectures}

Lemma 3.1 shows that generalized transformation $a^{2}+2 \cos (\pi / p) a b+b^{2}$ appears as a flip in the presence of an orbifold point of order $p$. The generalized cluster algebra constructed in this way is a subalgebra of a bigger standard cluster algebra (maybe of infinite rank) associated with triangulated surface while generalized exchange relation are sequences of standard mutations. Note that the positivity of Laurent polynomials for cluster algebras associated with bordered surfaces is known by 23 . This implies the positivity of Laurent polynomials in generalized cluster algebra associated with triangulations of the surface with arbitrary orbifold points.

We formulate the following conjecture.

Conjecture 5.1. If $\rho$ is a reciprocal polynomial with positive coefficients then any cluster variable of a generalized cluster algebra is expressed as a positive Laurent polynomial in the initial cluster.

We checked by direct inspection that the statement holds for finite type rank 2 cluster algebras.

The example above leads to a natural question:

Question: Is any generalized cluster algebra a subalgebra of some standard cluster algebra?

\section{REFERENCES}

[1] F. Bonahon, Shearing hyperbolic surfaces, bending pleated surfaces and Thurston's symplectic form, Ann. Fac. Sci. Toulouse Math 65 (1996), 233-297.

[2] Chekhov L., Teichmüller theory of bordered surfaces, SIGMA Symmetry Integrability Geom. Methods Appl., 3 (2007) Paper 066, 37 pp. (electronic).

[3] Chekhov L. O., Riemann surfaces with orbifold point, Proc. Steklov Math. Inst., 266 (2009), pp.1-26

[4] Chekhov L. O., Orbifold Riemann surfaces and geodesic algebras, J. Phys. A: Math. Theor. 42 (2009) Paper 304007, 32 pp. (electronic)

[5] Chekhov L., Fock V., A quantum Techmüller space, Theor. and Math. Phys. 120 (1999), 1245-1259, http://arxiv.org/abs/math.QA/9908165math.QA/9908165.

Chekhov L., Fock V., Quantum mapping class group, pentagon relation, and geodesics, Proc. Steklov Math. Inst. 226 (1999), 149-163.

[6] Chekhov L.O., Mazzocco M., Isomonodromic deformations and twisted Yangians arising in Teichmüller theory, Advances Math. 226(6) (2011) 4731-4775, arXiv:0909.5350

[7] L.O.Chekhov and R.C. Penner, On quantizing Teichmuller and Thurston theories, in: Handbook on Teichmuller Theory, Vol.1 (IRMA Lectures in Mathematics and Physics, Vol.11), ed. A.Papadopoulos, IRMA Publ., Strasbourg, France 2007. pp.579-646.

[8] G. Dupont, F. Palesi, Quasi-cluster algebras from non-orientable surfaces, http://arXiv:1105.1560

[9] Fock V.V., Combinatorial description of the moduli space of projective structures, http://arxiv.org/abs/hep-th/9312193.

[10] Fock V.V., Dual Teichmüller spaces, http://arxiv.org/abs/dg-ga/9702018. 
[11] V. V. Fock and A. B. Goncharov, Dual Teichmüller and lamination spaces, Chapter 15 in: Handbook on Teichmuller Theory, Vol.1 (IRMA Lectures in Mathematics and Physics, Vol.11), ed. A.Papadopoulos, IRMA Publ., Strasbourg, France. pp.647-684; math.DG/0510312

[12] V. V. Fock and A. B. Goncharov, Moduli spaces of local systems and higher Teichmüller theory, Publ. Math. Inst. Hautes Études Sci. 103 (2006), 1211, math.AG/0311149 v4.

[13] Fomin S., Shapiro M., Thurston D., Cluster algebras and triangulated surfaces. Part I: Cluster complexes, Acta Math. 201 (2008), no. 1, 83-146.

[14] Felikson A., Shapiro M., Tumarkin P., Cluster algebras and triangulated orbifolds, math.CO:1111.3449

[15] S. Fomin and A. Zelevinsky, Cluster algebras I: Foundations, J. Amer. Math. Soc. 15(2) (2002) 497-529.

[16] S. Fomin and A. Zelevinsky, The Laurent phenomenon, Adv. in Appl. Math. 28 (2002), no. $2,119-144$

[17] S. Fomin and A. Zelevinsky, Cluster algebra II: : Finite type classification Invent. Math., 154 (2003), no. 1, 63-121.

[18] Goldman W.M., Invariant functions on Lie groups and Hamiltonian flows of surface group representations, Invent. Math. 85 (1986), 263-302.

[19] Gekhtman M., Shapiro M.,Vainshtein A. Cluster algebras and Poisson geometry. Dedicated to Vladimir Igorevich Arnold on the occasion of his 65th birthday. Mosc. Math. J., 3 (2003), no. 3, 899-934, 1199.

[20] Gekhtman M., Shapiro M.,Vainshtein A. Cluster algebras and Weil-Petersson forms, Duke Math. J., 127 (2005), no. 2, 291-311.

[21] Th.Lam, P.Pylyavskyy, Laurent phenomenon algebras arXiv:1206.2611

R. M. Kaufmann, R. C. Penner, Closed/open string diagrammatics, Nucl. Phys. B748 (2006) $335-379$

[22] R. M. Kaufmann, R. C. Penner, Closed/open string diagrammatics, Nucl. Phys. B748 (2006) 335-379.

[23] Musiker G., Schiffler, R., Williams, L., Positivity for cluster algebras from surfaces, Adv. Math. 227 (2011), no. 6, 2241-2308.

[24] Penner R.C., The decorated Teichmüller space of Riemann surfaces, Comm. Math. Phys. 113 (1988), 299-339.

[25] W. P. Thurston, Minimal stretch maps between hyperbolic surfaces, preprint (1984), math.GT/9801039 